Check for updates

Cite this: Mater. Adv., 2021, 2, 7413

Received 4th August 2021

Accepted 21st August 2021

DOI: 10.1039/d1ma00689d

rsc.li/materials-advances

\title{
Morphological and mechanical characterization of high-strength sulfur composites prepared with variably-sized lignocellulose particles $\dagger$
}

\author{
Moira K. Lauer, ${ }^{a}$ Zoe E. Sanders, ${ }^{b}$ Ashlyn D. Smith ${ }^{\mathrm{ab}}$ and Rhett C. Smith (D)*a
}

\begin{abstract}
The extent to which lignocellulose biomass particle size influences the properties of biomass-sulfur composites prepared from these particles was evaluated. For this purpose several materials were prepared by the reaction of sulfur with peanut shell particles that had been fractionated into narrow size ranges using ASTM certified sieves. Eight particle size fractions with an upper cutoff range of 710 microns were thus used to prepare a series of eight composites PSS-1 to PSS-8. The use of biomass particles having defined size ranges allowed for a 36 -fold faster reaction rate relative to the analogous reactions employing unfractionated biomass, while the resultant composites still maintained excellent strength characteristics. Composites prepared with smaller biomass particles exhibited the most uniform dispersion, yet similar ultimate strength characteristics were observed for most of the composites irrespective of the biomass particle size. The strength characteristics of these materials could be rationalized by the interplay of the dispersion of filler in the network versus the unfavorable interactions between the hydrophilic biomass filler and hydrophobic sulfur network. This work highlights the importance of quantifying filler effect for microscopically non-homogeneous composite materials and provides insight on simple strategies for drastically impacting the time and energy expenditures for biomass composite synthesis and resultant properties.
\end{abstract}

\section{Introduction}

Developing mechanically-improved and more environmentally compatible structural materials is imperative to advance society beyond the petrochemical era. Concrete, comprised largely of ordinary portland cement (OPC), is the most widely-utilized construction material worldwide, with current production levels of over four and a half billion metric tons per year. ${ }^{1}$ The production of concrete accounts for about $30 \%$ of global materials utilization and is responsible for approximately $8 \%$ of anthropogenic $\mathrm{CO}_{2}$ production. ${ }^{1}$ The production of $\mathrm{CO}_{2}$ in the manufacture of concrete is unavoidable as the formation of its key intermediate, cement clinker, is made by heating mined carbonate minerals to $>1400{ }^{\circ} \mathrm{C}$ to form their respective oxides, a process that produces stoichiometric quantities of $\mathrm{CO}_{2}$. Cement production has approximately doubled in the last two decades and with an ever-increasing population and the rapid

\footnotetext{
${ }^{a}$ Department of Chemistry, Clemson University, Clemson, South Carolina, 29634, USA.E-mail: rhett@clemson.edu

${ }^{b}$ Department of Chemistry and Biology, Anderson University, Anderson,

South Carolina, 29621, USA

$\dagger$ Electronic supplementary information (ESI) available: Instrumentation, synthetic details, DSC curves, TGA curves, FTIR data, NMR spectral data, and material images. See DOI: 10.1039/d1ma00689d
}

modernization of less developed countries, global concrete production is forecast to continue to increase. ${ }^{2} \mathrm{~A}$ key component of efforts to reduce $\mathrm{CO}_{2}$ emissions must be to decrease the amount of OPC used in structural applications by adopting new formulations that use less environmentallydeleterious components. ${ }^{3-6}$ Although significant progress has been made towards this aim, many improved cement formulations still produce large amounts of $\mathrm{CO}_{2}$ while ultimately generating a non-recyclable product with a limited operational lifetime. Furthermore, many recent efforts have focused on filler-type additives that, while lowering the amount of new clinker that must be made, often do not impart attributes to their composite concretes that would improve their eventual recyclability, a property essential for a circular lifecycle. ${ }^{7-10}$

Our group has recently reported on various composite materials that have strength properties on par with or better than cement. These new composites are generally easily recycled, acid-resistant, and hydrophobic-characteristics that are possible due to the materials' high sulfur contents. ${ }^{11-33}$ These high sulfur content materials (HSMs) are prepared using waste sulfur that is produced in megaton quantities as a byproduct of fossil fuel refining. ${ }^{34,35}$ Mechanistically, such HSMs are predominantly prepared by inverse vulcanization. ${ }^{36-41}$ Inverse vulcanization is the crosslinking of olefin-containing 


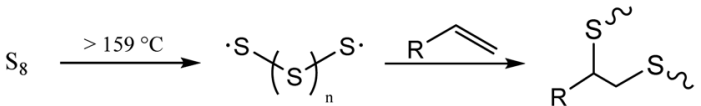

Scheme 1 Thermal homologation of $\mathrm{S}_{8}$ and its subsequent reaction with an olefin. When the $w t \%$ of sulfur is greater than that of the organic species, the process is known as inverse vulcanization.

organic species in concert with thermally-induced homologation of excess elemental sulfur (Scheme 1). Inverse vulcanization can be up to $100 \%$ atom economical and proceeds at significantly lower temperatures $\left(\sim 180^{\circ} \mathrm{C}\right)$ than that required for the production of cement clinker. The sulfur catenates in HSMs can homologate when the material is reheated, allowing the material to flow for facile remolding, pouring and paving. The HSMs can thus be reprocessed over many cycles with negligible changes in mechanical properties. Beyond structural applications, HSMs also have utility in lithium-sulfur batteries, ${ }^{42-50}$ as IR transparent lenses, ${ }^{51-57}$ and as adsorbents in environmental remediation. ${ }^{58-64}$ As the potential application scope of this class of materials continues to expand, important research on improved processing, recycling and alternative synthetic avenues to HSMs have been developed, even expanding the substrate scope to previously inaccessible organic monomers. ${ }^{39,65-72}$

Ideally HSMs will be as sustainably-produced as possible. To meet this goal the organic component to be reacted with waste sulfur must be renewably-sourced and preferably carbon negative to minimize or eliminate contributions to $\mathrm{CO}_{2}$ emission. Towards this end, researchers have demonstrated successful HSM production by reacting sulfur with fatty acids, ${ }^{25,31,33}$ terpenoids, ${ }^{19,21,57,73-75}$ starch, ${ }^{18,24}$ lignin, ${ }^{12,17,28,29}$ cellulose, ${ }^{13,19}$ and lignocellulosic biomass derivatives. ${ }^{16,17}$ Some of those biologically-derived materials do not possess the olefin functionalities needed for inverse vulcanization and therefore require derivatization or use of alternative reaction strategies. Derivatization, however, can detract from the atom economy and add to the economic/energetic cost of the process. Derivatizing biopolymers is also fraught with challenges due to biopolymer agglomeration or hornification during derivatization steps. ${ }^{76-86}$ Our previous work showed that if raw biomass is used as a precursor for the preparation of HSMs, the organic material must be mechanically milled to improve sulfur-organic surface area of contact and thus improve homogeneous dispersion of the material into resulting composites. Though lignocellulosic biomass is an especially attractive starting material, biomass faces a litany of well-known challenges to their commercial applications in competition with established petrochemicaland mineral-based materials. As a chemical feedstock, biomass can suffer from compositional changes depending on the source-even for the same species of origin-particle size/ shape effects, moisture content variation, and inhomogeneity of samples in terms of the percent composition of various plant components (stems, leaves, seed hulls, etc.) and thus variable chemical composition. Our previous work with peanut shell composites, for example, revealed that the composites' properties were strongly dependent on the peanut oil content of the peanut shell material employed in their preparation. ${ }^{16,17}$
The issues discussed above for biomass utilization necessitate assessing (1) the extent to which lignocellulose composite mechanical properties depend on particle size and (2) whether the chemical composition of the lignocellulosic feedstock varies on the basis of particle size due to the difference in grindability of divergent botanical structures of which the biomass is comprised. Chemical composition and particle morphology drastically impact particle dispersion and mechanical properties of composites prepared from cellulose nanocrystals, suggesting that these characteristics would also be important in lignocellulose-sulfur composites. ${ }^{87-90}$ Towards this aim, we have selected a model system of finely ground peanut shells as the biomass feedstock for the current study. Peanuts hold a fascinating place in the historical development of sustainable agriculture and chemistry dating to George Washington Carver's pioneering work on the use of peanuts as a nutrient-restoring plant for use in crop rotation-based land stewardship strategies. ${ }^{91-93}$ Later, peanut and soybean oil were some of the earliest feedstocks studied for preparation of what would later be called plastics, even predating the petrochemical plastics boom of the twentieth century. Peanutbased food products for human consumption are made primarily from the cotyledon (kernel) alone (Fig. 1), after it has been separated from the seed coat and shell components. Animal bedding and feed products can be made from some of the peanut shell waste, but the amount of shell waste available outpaces demand for these products, and smaller particle size fractions of peanut shells are not suitable for use in these applications. As a result, large quantities of bulk shell waste and small particle-size waste remain to be valorised. Peanuts remain a high production crop with annual production of about $44 \mathrm{Mt}$, of which over $11 \mathrm{Mt}$ is in the form of shells that are not nutritionally valuable for human consumption. ${ }^{94}$ Peanut shells are primarily comprised of cellulose (52\%) and lignin (35\%) with $\sim 1 \mathrm{wt} \%$ being peanut oil.

In previous work, we demonstrated several strategies for preparing composites from peanut shell and sulfur waste. The properties of these composites are competitive with traditional structural materials like OPC, suggesting their possible

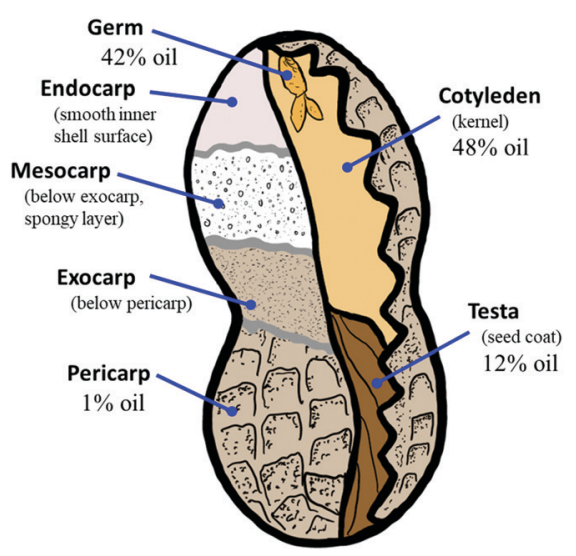

Fig. 1 Botanical breakdown of a typical two-seed peanut legume with oil content for peanut-oil-containing components. Artist's rendering based on information accessed 4-24-21 at www.aboutpeanuts.com/peanutfacts/97-the-parts-and-composition-of-the-peanut. 
commercial applicability. Those studies demonstrated that high strength materials are possible due to the presence of a small amount of olefin-containing peanut oil ( $\sim 1 \%$ by weight) in the peanut shells, but only one particle size was used. The extent to which mechanical properties may also be attributable to a filler effect that depends on particle size of the lignocellulosic precursor thus remains unknown. ${ }^{95-102}$ Additionally, the material referred to as peanut shell waste can be further subdivided as being comprised of several botanical structures having different oil contents and therefore different potentials for crosslinking by inverse vulcanization (Fig. 1). It is conceivable that differences in the grindability of these botanical structures might influence their distribution by particle size in ground peanut shell materials. Herein, peanut shells were fractionated into eight different size ranges with an upper cutoff of $710 \mu \mathrm{m}$ (Fig. 1 and Table 1) and the oil (olefin) content of each fraction was evaluated by ${ }^{1} \mathrm{H}$ NMR spectrometric analysis. Each fraction was reacted with elemental sulfur to produce eight HSMs (PSS1-8). The extent to which particle size influences resultant composite mechanical properties were assessed by mechanical strength analysis, while morphology and thermal behavior were assessed by, differential scanning calorimetry (DSC), scanning electron microscopy element mapping by energy-dispersive X-ray analysis (SEM-EDX), and thermogravimetric analysis (TGA).

\section{Results and discussion}

\section{Characterization and fractionation of biomass feedstock}

The initial peanut shell material used for the current study was a finely-ground material (Fig. 2, unfractionated) from a facility that produces animal feed products from peanut shells. Animal feed production can only utilize a fraction of total peanut shells production and after processed into animal feed, there is a fraction remaining that is not usable for animal feed products due to the small particle size. This small particle size is, however, especially amenable to the target process of converting the shell residue into homogeneous composites. The industrial peanut shell product was therefore further fractionated using ASTM standard sieves beginning by removing particles larger than $710 \mu \mathrm{m}$, a portion that represented a negligible fraction of the total mass. The fractionation process was continued by collecting the material that would not pass through the $500 \mu \mathrm{m}$ sieve to yield

Table 1 Peanut shell fractionation data

\begin{tabular}{lll}
\hline Fraction code & $\begin{array}{l}\text { Minimum size } \\
\text { cutoff }(\mu \mathrm{m}) / \text { ASTM size }\end{array}$ & $\begin{array}{l}\text { Maximum size cutoff } \\
(\mu \mathrm{m}) / \text { ASTM size }\end{array}$ \\
\hline PS-1 & $500 / 35$ & $710 / 25$ \\
PS-2 & $300 / 50$ & $500 / 35$ \\
PS-3 & $250 / 60$ & $300 / 50$ \\
PS-4 & $212 / 70$ & $250 / 60$ \\
PS-5 & $150 / 100$ & $212 / 70$ \\
PS-6 & $125 / 120$ & $150 / 100$ \\
PS-7 & $75 / 200$ & $125 / 120$ \\
PS-8 & $\mathrm{NA}^{a}$ & $75 / 200$
\end{tabular}

${ }^{a}$ All particles passing the ASTM 200 sieve were retained in this sample. material with particles having dimensions of $500-710 \mu \mathrm{m}$ (fraction PS-1). Sequentially smaller size ASTM sieves were employed according to the workflow provided in Table 1 to yield seven additional fractions from PS-2 through the smallest particle size fraction PS-8 (Fig. S1, ESI $\dagger$ ). Optical micrographs of PS-X fractions (Fig. 2) demonstrate the range of sizes resulting from the fractionation process as well as revealing the irregular particle shapes present in the materials.

Due to inhomogeneity and small sample sizes analysed in DSC runs $(\sim 5 \mathrm{mg})$, the magnitude and position of $T_{\mathrm{g}}$ values varied slightly for duplicate analysis of each PS- $\boldsymbol{X}$ fraction. The position of the $T_{\mathrm{g}}$ attributable to cellulose varied, on average, by $7{ }^{\circ} \mathrm{C}$ while the $T_{\mathrm{g}}$ values attributable to the lignin fraction of the peanut shell varied, on average, by $6{ }^{\circ} \mathrm{C}$ (Fig. S2-S8, ESI $\dagger$ ). Unsurprisingly, all fractions of peanut shells had nearly indistinguishable infrared (IR) spectra (Fig. S9, ESI $\dagger$ ) but somewhat different TGA results. All peanut shell fractions show an initial mass loss of $\sim 9 \mathrm{wt} \%$ due to adsorbed moisture in the range $25-120{ }^{\circ} \mathrm{C}$ (Fig. 3A). Due to increased surface area, smaller peanut shell particles began to decompose at slightly lower temperatures and much more rapidly than larger particle sizes while also burning more completely to give a lower char yield (i.e., less residue at $800{ }^{\circ} \mathrm{C}$, Fig. 3B). Notable differences in thermal stability are observed only at temperatures in excess of $300{ }^{\circ} \mathrm{C}$, as obviated in the derivative TGA inset shown in Fig. 3C. At the composite preparation temperature of $180{ }^{\circ} \mathrm{C}$, there should thus be no particle size-dependant thermal degradation that could influence composite properties.

\section{Composite synthesis and chemical characterization}

Each PS- $\boldsymbol{X}$ fraction (10 wt $\%$ ) was mixed with sulfur (90 wt \%) and the mixture was then heated at $180{ }^{\circ} \mathrm{C}$ while stirring until the mixture became visibly homogeneous to prepare eight inversely vulcanized materials PSS- $\boldsymbol{X}$, where $X$ corresponds to number of PS- $\boldsymbol{X}$ fraction used for the composite's preparation (Table 1). The feed composition of $10 \mathrm{wt} \%$ biomass and $90 \mathrm{wt} \%$ sulfur was selected to match that used to prepare previously-reported $\mathbf{P S}_{\mathbf{9 0}}$, which was prepared from the unfractionated form of the same peanut shells used for the current study. PS $_{\mathbf{9 0}}$ also possessed compressional strength characteristics on par with that required for residential concrete construction and so represents a benchmark for comparing properties to composites prepared from fractionated peanut shells.

While unfractionated peanut shells required three days of reaction to reach homogeneity in the preparation of $\mathbf{P S}_{\mathbf{9 0}}$, fractionated shells were found to homogenize significantly more quickly during reaction, requiring only $2 \mathrm{~h}$ total reaction time. Although all eight of the reaction mixtures became homogenous at the reaction temperature, the two materials that were prepared with the largest particle-size fractions (PSS-1 and PSS-2) phase separated on cooling (Fig. S10, ESI $\dagger$ ). Only homogeneous materials PSS-3 through PSS-8 will be further discussed. Cylinders of PSS-3-8 suitable for compressional analysis were prepared by remelting and pouring the molten material into silicone moulds (Fig. 4). In order to better understand the impact of filler morphology on ultimate composite 
A)

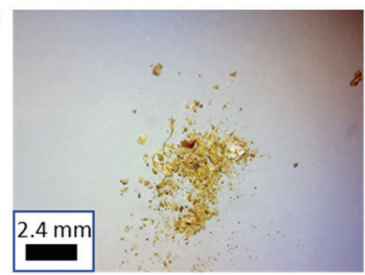

Unfractionated

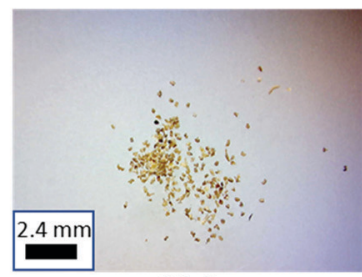

PS-6

B)

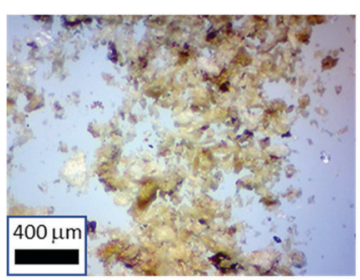

Unfractionated

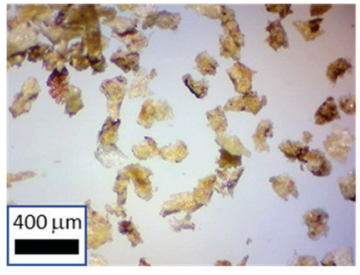

PS-6

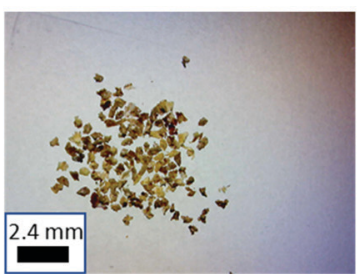

PS-3

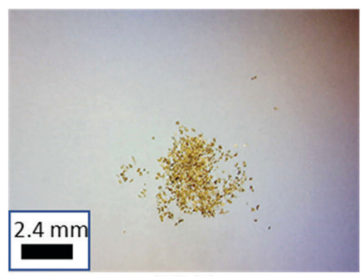

PS-7

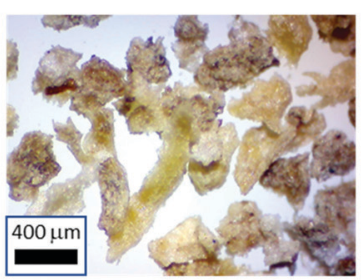

PS-3

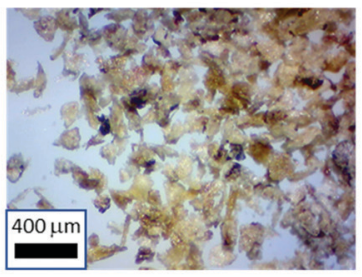

PS-7

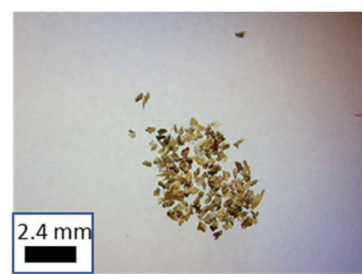

PS-4

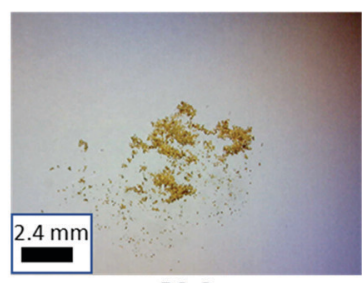

PS-8

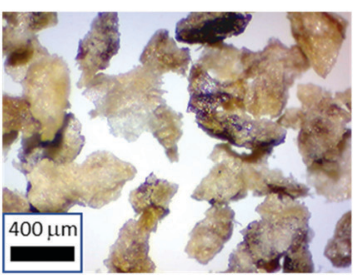

PS-4

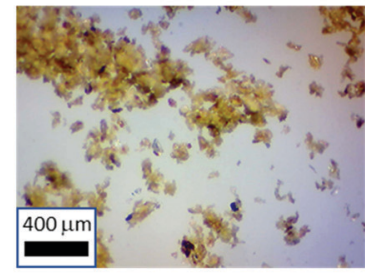

PS-8

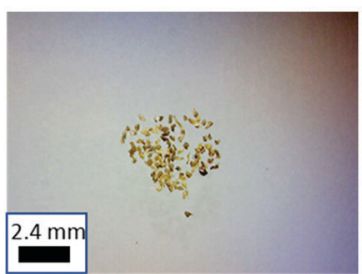

PS-5

Fig. 2 Optical micrographs demonstrating the range of particle sizes (A) and irregular particle shapes (B) in peanut shell fractions PS- $\boldsymbol{X}$

properties, two control materials were prepared (Fig. 4B and C). The first control material was prepared to attempt to understand the impact of the peanut shells as filler on material properties. This was done by preparing a material containing no biomass and instead containing only sulfur and peanut oil in the same ratios as would be found in a material containing $10 \%$ peanut shell in which $1 \%$ of that mass was attributable to peanut oil. The second control material sought out to understand the impact of the peanut oil's incorporation into the lignocellulosic biomass as well as the impact of lignin-cellulose crosslinks. This material was consequently prepared with the same amount of peanut oil in the first control material but also containing a mixture of microcrystalline cellulose and alkali lignin in a $3: 2$ ratio (the approximate ratio found in the peanut shell) as $10 \mathrm{wt} \%$ of the feed ratio. Both of these materials would not homogenize and showed drastic phase separation upon cooling in addition to a light-yellow colour indicative of (non-inversely vulcanized) orthorhombic sulfur. The inability for these materials to homogenize emphasizes the importance of the native biomass structure in facilitating the inverse vulcanization reaction while uniformly dispersing the filler.
Because metastable polymeric sulfur has the potential to slowly revert to $S_{8}$ over time when present in a crosslinked network, all materials were cured at room temperature for sixteen days before any mechanical, thermal, or morphological analysis was performed to ensure the probing of end-use structure and properties. ${ }^{13,16,17,103}$

HSMs can vary in the extent to which monomer feed sulfur is incorporated into their structures. Elemental analysis revealed that this holds for PSS3-8 as well, with the composition of each materials containing somewhat less than $90 \%$ of sulfur ( 81-88 wt\%, Fig. 5A). A plot of particle size versus percent incorporation of sulfur revealed a definite trend in sulfur incorporation as a function of particle size, with composites prepared from the smallest and largest particles incorporating the most sulfur (86-88 wt $\%$ ) and the intermediary composite PSS-5 incorporating the least sulfur $(\sim 81 \mathrm{wt} \%$, Fig. 4). To better understand this trend, extraction of composites PSS- $\boldsymbol{X}$ was undertaken utilizing $\mathrm{CS}_{2}$. Orthorhombic sulfur-sulfur that is not covalently linked to peanut shell molecules-is quite soluble in $\mathrm{CS}_{2}$, while peanut shell and covalently-attached sulfur is completely insoluble (as confirmed by elemental microanalysis 


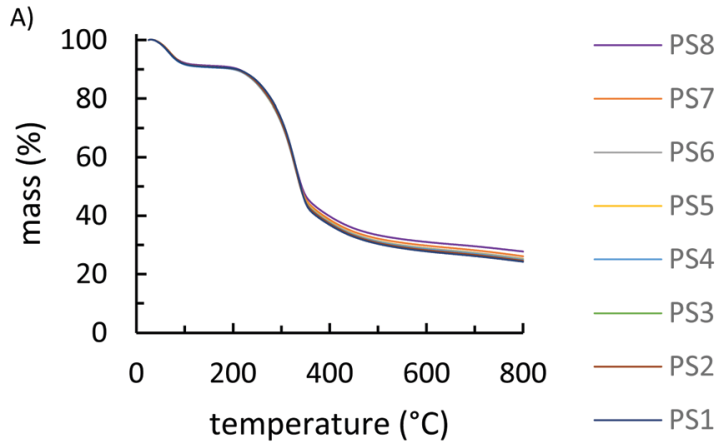

B)
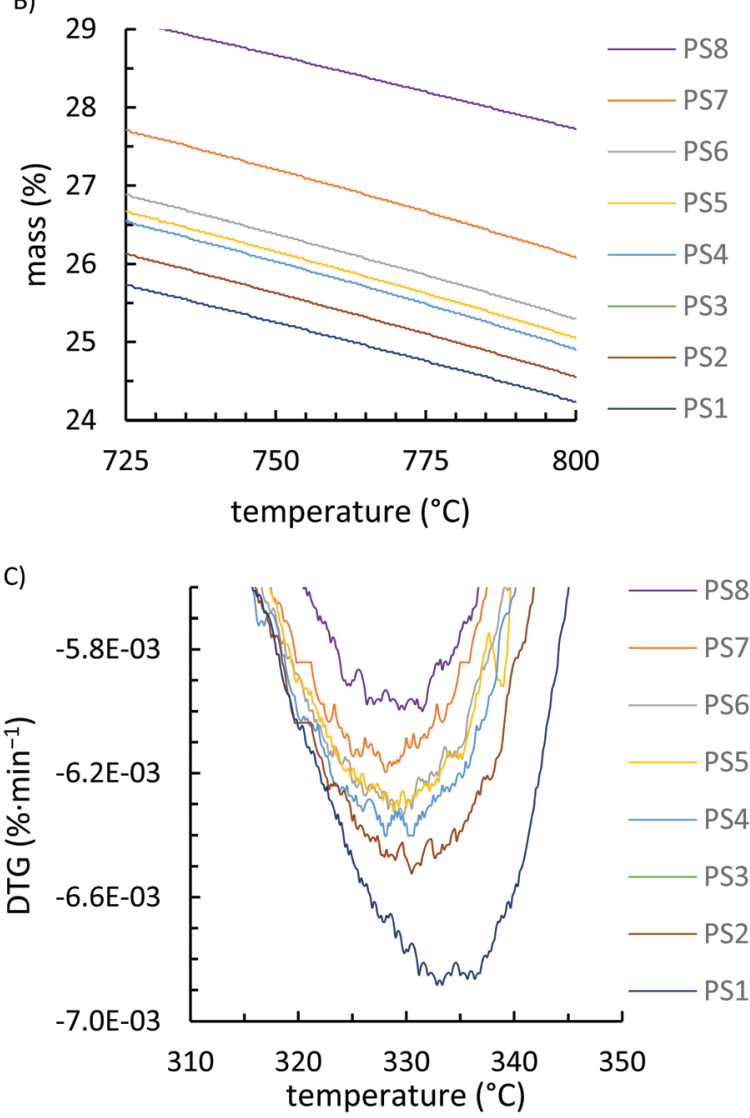

Fig. 3 Thermogravimetric analysis of PS- $\boldsymbol{X}$ fractions from $25-800{ }^{\circ} \mathrm{C}$ (A), from $725-800{ }^{\circ} \mathrm{C}$ to emphasize the difference in residual mass (B), and the derivative TGA (DTG) curve over the primary decomposition step, emphasizing the more rapid and lower-temperature decomposition with smaller particle size (C). Note that PS-2 and PS-3 are coincident.

of extracts). Fractionation of the material with $\mathrm{CS}_{2}$ paired with elemental analysis results therefore allows for the determination of how much of the sulfur present in each material is free or covalently bound to peanut shells. The fraction of each material that was soluble in $\mathrm{CS}_{2}$ varied predictably with the amount of sulfur that was incorporated into the material and, with the exception of PSS-8, the trend in total incorporated sulfur tracked with the amount of sulfur covalently bound (Fig. 5B). Materials PSS-4, PSS-5, and PSS-6 were effectively unable to crosslink with sulfur resulting in the largest discrepancy between feed ratio and the amount of incorporated sulfur whereas PSS-3, PSS-7, and
A)
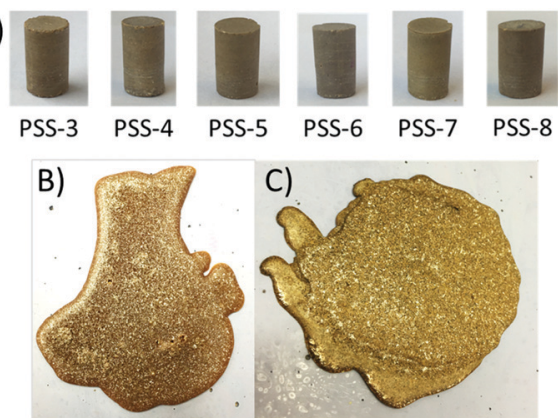

Fig. 4 Photos of compression test cylinders (A) and identically-prepared control materials made by reaction of sulfur and peanut oil (B) or sulfur, peanut oil, lignin, and cellulose $(C)$ in the same ratios present in peanut shells used to prepare PSS-3-8. Control materials are heterogeneous and within minutes of cooling begin to rapidly re-develop the lighter yellow colour typical of orthorhombic sulfur, while the brown-orange colour of PSS- $\boldsymbol{X}$ composites persists for over 16 days (aging time before analysis herein).

A)
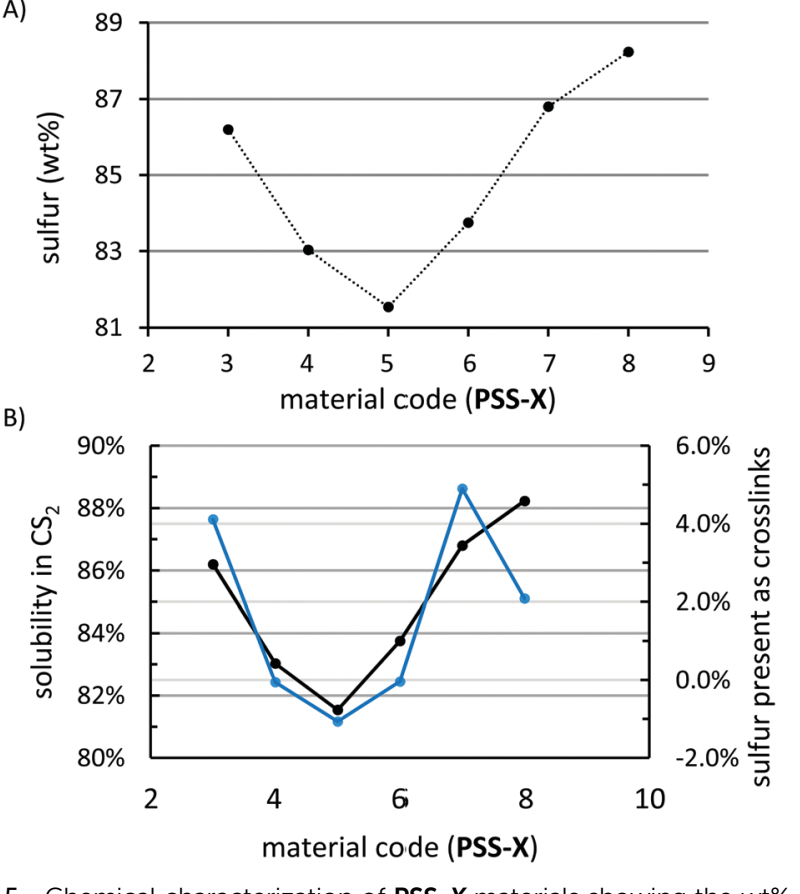

Fig. 5 Chemical characterization of PSS- $\boldsymbol{X}$ materials showing the wt\% of the material that was determined to be sulfur by elemental microanalysis (A), the amount of orthorhombic sulfur that was determined to be present in the material by CS2 fractionation studies ( $B$, left axis, black trace) and the amount of sulfur that was determined to be present in the composite as crosslinked sulfur (the difference between the amount of sulfur present and the amount of orthorhombic sulfur identified) (B, right axis, blue trace).

PSS-8 were able to crosslink some amount of sulfur resulting in good agreement between the feed ratio and amount of sulfur incorporated into the material. The outlier, PSS-8 was able to distribute peanut shell particles most effectively through the sulfur network (from SEM analysis, vide infra), likely due to the low viscosity of the melt resulting from the small particle size. These data indicate that homogenization of organic species with 
few crosslinkable sites may be benefited by using as small a particle size as possible to provide good dispersion in the material.

\section{Morphological characteristics of biomass-sulfur composites}

To gain further insight into the morphology of the materials, the composites were examined by scanning electron microscopy element mapping by energy-dispersive X-ray analysis (SEM-EDX, Fig. 6). Whereas composites prepared from smaller particle-size biomass showed homogeneous distribution of organic material throughout the HSM (i.e., PSS-7 and PSS-8, Fig. 6I-L), organic particle agglomeration became more pronounced for the materials prepared with larger particle size precursors, as evident from the images of PSS-3 and PSS-4 (Fig. 6A-D). Such trends in particle dispersion as a function of their size is a common phenomenon in composite formation that can often be traced to differences in viscosity. ${ }^{104}$ In the current case, the increased melt viscosity is likewise evident when pouring molten samples of composites with larger particle size precursors.

Thermal analysis revealed little in the differences between composites. TGA analysis of PSS- $\boldsymbol{X}$ showed a similar trend as observed for PS- $\boldsymbol{X}$ but on a smaller magnitude commensurate with the lower quantity of organics in the composites (Fig. S11, ESI $\dagger$ ). Results obtained by DSC showed nearly identical curves for all of the materials except that PSS-3 showed a consistent (triplicate analysis) cold crystallization exotherm at $\sim 20{ }^{\circ} \mathrm{C}$ (Fig. S12-S17, ESI $\dagger$ ). This can be attributed to the poor dispersion of peanut shells in this material producing a more rigid network in local areas, hindering crystallization on cooling, thus resulting in partial recrystallization upon reheating. The materials also showed a linear trend in crystallization temperature with the exception of PSS-4 that showed a surprisingly high crystallization temperature consistent with the lack of crosslinking observed (see also Table S3 and Fig. S18, ESI $\dagger$ ).

\section{Composite mechanical strength and correlation with botanical composition/fat content}

Compressive strength measurements were conducted in an attempt to find a correlation between strength and particle size. Previously reported PS $_{\mathbf{9 0}}$, prepared from $90 \mathrm{wt} \%$ sulfur and unfractionated peanut shells, exhibited an impressive compressive strength of $21.3 \pm 1.2 \mathrm{MPa}{ }^{16,17,103}$ A higher average compressive strength was observed for all of the PSS-X composites that were prepared from fractions of the same peanut shells, but the compressive strength was less reproducible from sample-to-sample for PSS- $\boldsymbol{X}$ compared to $\mathbf{P S}_{\mathbf{9 0}}$, as reflected in the larger standard deviations shown for these data in Table 2 (all measurements were done in triplicate). Unfortunately, even with replicate analysis, most materials had such a large standard deviation that the materials could not be differentiated in terms of compressional strength as a function of particle size.

The effect of fillers on a material's mechanical properties can generally be described by two primary considerations: (1) the compatibilization between the filler and the matrix, and (2) the dispersion of the filler in the matrix. ${ }^{101,104}$ For the PSS- $\boldsymbol{X}$
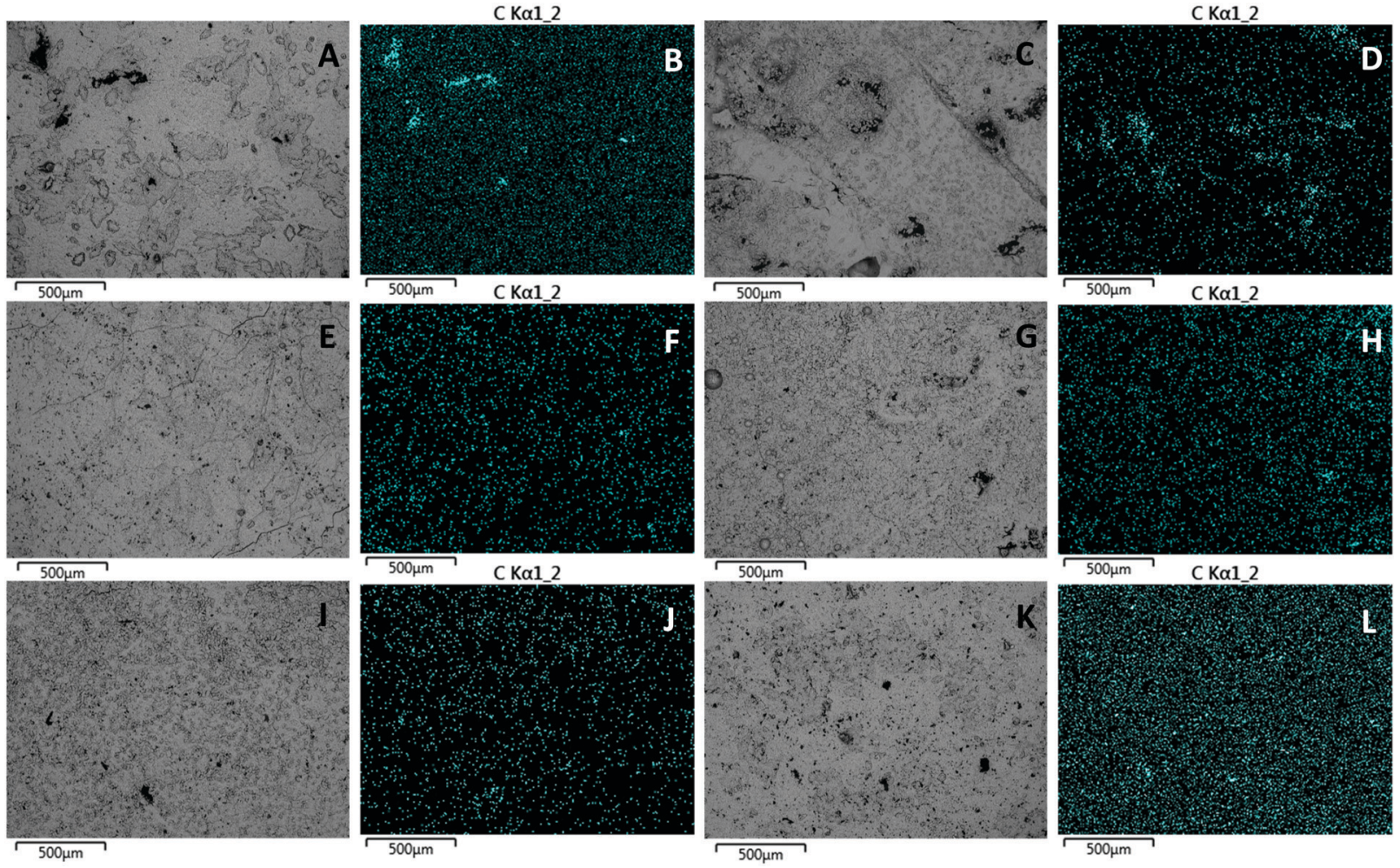

Fig. 6 SEM-EDX images of PSS- $X$ showing the EM image (black and white: A, C, E, G, I and K) and the carbon element mapping (blue images: B, D, F, H, J and L) for PSS-3 (A and B), PSS-4 (C and D), PSS-5 (E and F), PSS-6 ( $G$ and H), PSS-7 (I and J), and PSS-8 (K and L). 
Table 2 Mechanical properties of materials PSS- $\boldsymbol{X}$ with the amount of hexane soluble material found to be contained in the peanut shell fractions PS- $X$

\begin{tabular}{llll}
\hline Material & Strength $^{a}(\mathrm{MPa})$ & $n^{b}$ & $\begin{array}{l}\text { wt\% of hexane soluble in } \\
\text { corresponding PS- } \boldsymbol{X}(\%)\end{array}$ \\
\hline PSS-3 & $29.2 \pm 6.3$ & 4 & 1.10 \\
PSS-4 & $30.6 \pm 0.9$ & 3 & 0.00 \\
PSS-5 & $28.0 \pm 3.2$ & 4 & 0.50 \\
PSS-6 & $30.3 \pm 10.2$ & 5 & 0.04 \\
PSS-7 & $24.3 \pm 4.4$ & 4 & 0.54 \\
PSS-8 $_{\text {PS }^{c}{ }^{c}}^{25.4}$ & $21.3 \pm 10.6$ & 8 & 0.05 \\
& & 3 & 1.60
\end{tabular}

${ }^{a}$ Material ultimate strength under a compressional force. ${ }^{b}$ The number of replicates performed to obtain the strength and standard deviation. ${ }^{c}$ The material prepared in our previous manuscript from unfractionated peanut shells and heated for three days.

series of materials, the compatibility is not favourable due to the highly hydrophilic nature of the peanut shells juxtaposed with the hydrophobic nature of sulfur. On this basis, maximizing surface area (i.e., using smaller particle size filler) should be unfavourable for the development of strength properties. This is in contrast to the trend seen with particle dispersion where better dispersion was generally observed with smaller particle size. These two counterbalancing trends are likely responsible for the relatively similar strength properties across the series.

Based on the results from $\mathrm{CS}_{2}$ extractions, it may have been anticipated that PSS-3, PSS-7, and PSS-8 would have the greatest strength due to the peanut shell not only acting as a filler but also crosslinking with sulfur. Such a supposition is also borne out in strength trends of particle-free HSMs, wherein mechanical strength generally increases with increased covalent crosslinking. Such a trend was not observed in the case of composites PSS- $\boldsymbol{X}$, however. The strengths of materials on either extreme of particle size were not as reproducible as are strengths of particlefree HSMs. The underlying cause for this behaviour in PSS-3 was traced to significant agglomeration of organic material observed in the SEM images. In contrast, the dispersion of particles in PSS-8 was much higher, though unfavourable sulfur-biomass interface effects are maximized among members of the series, so that PSS-8 also shows diminished strength. The other materials exhibited some extent of sulfur crosslinking, and consequently samples PSS-7 had a relatively reproducible strength yet provided a relatively low average compressional strength value. The reproducibility can be attributed to the uniform dispersion of organic particles through the sulfur matrix-the best seen throughout the series-providing consistent samples for analysis. The source of low strength can again be rationalized by the high surface area of the very small particles.

The materials that exhibited very little sulfur crosslinkingPSS-4, PSS-5, and PSS-6-produced surprisingly high average strength values. In these cases, the peanut shells are primarily acting as a filler with low contribution to a crosslinked network to stabilize polymeric sulfur. In this morphological regime, larger particle size produces the most reproducible results indicating minimization of surface area was more beneficial than the uniform dispersion of peanut shell.
The property variability and potential relationship to the complex interplay of surface area and size effects motivated us to quantify the amount of peanut oil in each of the peanut shell fractions. By stirring the peanut shells in hexane overnight, the peanut oil was extracted from the shell fractions, isolated, and quantified by ${ }^{1} \mathrm{H}$ NMR spectrometry with an internal standard (2,3,4,5,6-pentafluorobenzaldehyde). This fractionation process resulted in removal of peanut oil from the shells (Table 2). The amount of oil extractable from the peanut shell samples varied across the series. Unfortunately, the presence of unidentified compounds other than peanut oil that produced resonances in the NMR spectrum made it difficult to make any conclusive deductions from these data for most of the samples (Fig. S19 and S20, ESI $\dagger$ ). The ${ }^{1} \mathrm{H}$ NMR data did, however, help to explain the wide variability of PSS-6 sample compressive strengths. In agreement with the $\mathrm{CS}_{2}$-extraction study that indicated that PSS-6 crosslinked a negligible amount of sulfur, PSS-6 also contained trivial quantities of peanut oil or any other hexane-soluble species. These data suggest that PSS-6 features the poorest compatibility/covalent crosslinking between peanut shell particles and sulfur among all of the composites studied. All materials possessed considerably less hexane soluble material than unfractionated peanut shell, a result that made practical sense but seemed counterintuitive considering the PSS- $\boldsymbol{X}$ series exhibited slightly better strength characteristics than $\mathbf{P S}_{\mathbf{9 0}}$. This result may be explained by the difference in requisite heating times. The longer heating times required for $\mathbf{P S}_{\mathbf{9 0}}(72 \mathrm{~h}$ versus $2 \mathrm{~h}$ for PSS- $\boldsymbol{X})$ likely results in some destruction of the native peanut shell structure which we have previously shown to be disadvantageous in cellulose systems. ${ }^{19}$ As previously noted, attempts to prepare composites analogous to $\mathbf{P S}_{\mathbf{9}}$ by heating sulfur with unfractionated peanut shells for shorter times led to visibly heterogeneous materials, so a direct comparison of PSS- $\boldsymbol{X}$ to that hypothetical direct control material is not possible. Fractionation thus proved to be successful in reducing reaction time, thereby preserving the native structure of the lignocellulosic components, and reducing energy requirements for composite preparation, but at the expense of material homogenization and reproducibility of material properties.

\section{Conclusions}

This work demonstrates the ability to attain high strength materials from unfractionated, unmodified biomass after heating to a relatively low temperature for only a few hours using raw biomass that has been fractionated by a simple mechanical sifting process. All prepared materials possessed compressional strength characteristics on par with residential Portland cement. Opposing trends in the minimization of surface area and the more uniform dispersion of biomass filler provides rationale for similarity of strength between all of the materials in the series. Fractionation of biomass into narrow particle size distributions did, however, prove to be beneficial for significantly decreasing reaction times and thus energy costs for achieving the high-strength materials. 


\section{Experimental}

\section{General considerations}

Fourier transform infrared spectra were obtained using an IR instrument (Shimadzu IRAffinity-1S) with an ATR attachment. Scans were collected over the range $400-4000 \mathrm{~cm}^{-1}$ at ambient temperature with a resolution of 8. TGA was recorded (Mettler Toledo TGA 2 STARe System) over the range $20-800{ }^{\circ} \mathrm{C}$ with a heating rate of $10{ }^{\circ} \mathrm{C} \mathrm{min}^{-1}$ under a flow of $\mathrm{N}_{2}\left(100 \mathrm{~mL} \mathrm{~min}^{-1}\right)$. Each measurement was acquired in duplicate and presented results represent an average value. DSC was acquired (Mettler Toledo DSC 3 STARe System) over the range 60 to $150{ }^{\circ} \mathrm{C}$ with a heating rate of $5{ }^{\circ} \mathrm{C} \mathrm{min}^{-1}$ under a flow of $\mathrm{N}_{2}\left(200 \mathrm{~mL} \mathrm{~min}{ }^{-1}\right)$. Each DSC measurement was carried out over three heat-cool cycles. Each measurement was acquired in triplicate to ensure consistent results were obtained.

Carbon disulfide extractions were performed by suspending $0.3 \mathrm{~g}$ of finely ground material (measured to $0.0001 \mathrm{~g}$ ) in $20 \mathrm{~mL}$ of $\mathrm{CS}_{2}$, allowing the solid to settle for 30 minutes, pipetting off the supernatant into a separate vial, and adding another $20 \mathrm{~mL}$ of $\mathrm{CS}_{2}$. This process was repeated an additional 3 times so that a total of 5 washes was performed. The residual $\mathrm{CS}_{2}$ was evaporated under a flow of $\mathrm{N}_{2}$ and each vial was weighed to determine the fraction that was soluble (collected as supernatant) or insoluble (remained in the initial vial).

Compressional analysis was performed on a Mark-10 ES30 test stand equipped with a M3-200 force gauge (1 kN maximum force with $1 \mathrm{~N}$ resolution) with an applied force rate of 3-4 $\mathrm{N} \mathrm{s}^{-1}$. Compression cylinders were cast from silicone resin moulds (Smooth-On Oomoo 30 tin-cure) with diameters of approximately $6 \mathrm{~mm}$ and heights of approximately $10 \mathrm{~mm}$. Samples were manually sanded to ensure uniform dimensions and measured with a digital calliper with $0.01 \mathrm{~mm}$ resolution. Compressional analysis was initially performed three times; however, more samples were analysed when the standard deviation was greater than four MPa. The number of replicates was increased until the standard deviation was consistent with additional runs. The number of replicates for each sample is reported in Table 2 .

SEM was acquired on a Schottky Field Emission Scanning Electron Microscope SU5000 operating in variable pressure mode with an accelerating voltage of $15 \mathrm{keV}$.

\section{Materials and methods}

Ground peanut shells were obtained from Golden Peanut and Tree Nuts (Product ES). Sulfur was obtained from Alfa Aesar. Carbon disulfide was obtained from Beantown Chemical. 2,3,4,5,6-Pentafluorobenzaldehyde was obtained from Acros Organics. All reagents were used as received unless otherwise specified.

\section{General composite synthesis}

PSS- $\boldsymbol{X}$ materials were prepared by combining $9.0 \mathrm{~g}$ of sulfur and $1.1 \mathrm{~g}$ of fractionated peanut shells in a glass vial equipped with a Teflon coated stir bar. The vials were placed in an oil bath set to $180{ }^{\circ} \mathrm{C}$ for two hours. Specific reaction conditions can be found in the ESI. $\dagger$

\section{Hexane extraction and NMR analysis}

Peanut shell powder $(8 \mathrm{~g})$ was suspended in hexane $(150 \mathrm{~mL})$ and stirred at room temperature for $24 \mathrm{~h}$. The suspension was filtered, and the hexane was removed from the filtrate under reduced pressure. The yield of material was determined as a first approximation of peanut oil in each fraction. A solution of 2,3,4,5,6-pentaflourobenzaldehyde (PFBA) in $\mathrm{CDCl}_{3}$ was prepared by serial dilution. First, $0.4657 \mathrm{~g}$ of PFBA was dissolved in $9.5337 \mathrm{~g}$ of $\mathrm{CDCl}_{3}, 2.0118 \mathrm{~g}$ of this solution was then dissolved in $18.0001 \mathrm{~g}$ of $\mathrm{CDCl}_{3}$ to bring the final concentration of the solution to $4.6817 \mathrm{mg}$ of PFBA per $\mathrm{g}$ of $\mathrm{CDCl}_{3}$. Approximately one gram of this solution was used to dissolve the residue for each hexane soluble fraction and then this solution was transferred to an NMR tube for analysis. External standards were also prepared by dissolving 10,20 , or $30 \mathrm{mg}$ of peanut oil into $1 \mathrm{~g}$ of $\mathrm{CDCl}_{3}$.

\section{Conflicts of interest}

There are no conflicts to declare.

\section{Acknowledgements}

Funding for this project from the National Science Foundation (CHE-1708844) is gratefully acknowledged.

\section{Notes and references}

1 K. L. Scrivener, V. M. John and E. M. Gartner, Cem. Concr. Res., 2018, 114, 2-26.

2 R. M. Andrew, Earth Syst. Sci. Data, 2018, 10, 195-217.

3 K. L. Scrivener, V. M. John and E. M. Gartner, Eco-efficient cements: Potential, economically viable solutions for a low$\mathrm{CO}_{2}$ cement-based materials industry, Paris, 2016.

4 S. Shirani, A. Cuesta, A. Morales-Cantero, A. G. De la Torre, M. P. Olbinado and M. A. G. Aranda, Cem. Concr. Res., 2021, 147, 106499.

5 R. Zhao, L. Zhang, G. Fan, Y. Chen, G. Huang, H. Zhang, J. Zhu and X. Guan, Cem. Concr. Res., 2021, 144, 106420.

6 Y. Briki, F. Avet, M. Zajac, P. Bowen, M. B. Haha and K. Scrivener, Cem. Concr. Res., 2021, 146, 106477.

7 A. Al-Hamrani, M. Kucukvar, W. Alnahhal, E. Mahdi and N. C. Onat, Materials, 2021, 14, 351.

8 F. Blomsma and G. Brennan, J. Ind. Ecol., 2017, 21, 603-614.

9 J. Deschamps, B. Simon, A. Tagnit-Hamou and B. Amor, J. Cleaner Prod., 2018, 185, 14-22.

10 Y. Yu, D. M. Yazan, S. Bhochhibhoya and L. Volker, J. Clean. Prod., 2021, 293, 126083.

11 T. Thiounn, M. K. Lauer, M. S. Bedford, R. C. Smith and A. G. Tennyson, $R S C$ Adv., 2018, 8, 39074-39082. 
12 M. S. Karunarathna, M. K. Lauer, T. Thiounn, R. C. Smith and A. G. Tennyson, J. Mater. Chem. A, 2019, 7, 15683-15690.

13 M. K. Lauer, T. A. Estrada-Mendoza, C. D. McMillen, G. Chumanov, A. G. Tennyson and R. C. Smith, Adv. Sustainable Syst., 2019, 3, 1900062.

14 M. S. Karunarathna, M. K. Lauer and R. C. Smith, J. Mater. Chem. A, 2020, 8, 20318-20322.

15 M. S. Karunarathna, M. K. Lauer, A. G. Tennyson and R. C. Smith, Polym. Chem., 2020, 11, 1621-1628.

16 M. K. Lauer, M. S. Karunarathna, A. Tennyson and R. C. Smith, Mater. Adv., 2020, 1, 590-594.

17 M. K. Lauer, M. S. Karunarathna, A. G. Tennyson and R. C. Smith, Mater. Adv., 2020, 1, 2271-2278.

18 M. K. Lauer and R. C. Smith, Comprehensive Reviews in Food Science and Food Safety, 2020, pp. 1-53 , DOI: 10.1111/ 1541-4337.12627.

19 M. K. Lauer, A. G. Tennyson and R. C. Smith, ACS Appl. Polym. Mater., 2020, 2, 3761-3765.

20 C. V. Lopez, M. S. Karunarathna, M. K. Lauer, C. P. Maladeniya, T. Thiounn, E. D. Ackley and R. C. Smith, J. Polym. Sci., 2020, 58, 2259-2266.

21 C. P. Maladeniya, M. S. Karunarathna, M. K. Lauer, C. V. Lopez, T. Thiounn and R. C. Smith, Mater. Adv., 2020, 1, 1665-1674.

22 T. Thiounn, M. S. Karunarathna, L. M. Slann, M. K. Lauer and R. C. Smith, J. Polym. Sci., 2020, 58, 1347-1364.

23 T. Thiounn, M. K. Lauer, M. S. Karunarathna, A. G. Tennyson and R. C. Smith, Sustainable Chem., 2020, 1, 183-197.

24 M. K. Lauer, A. G. Tennyson and R. C. Smith, Mater. Adv., 2021, 2, 2391-2397.

25 A. D. Smith, T. Thiounn, E. W. Lyles, E. K. Kibler, R. C. Smith and A. G. Tennyson, J. Polym. Sci., Part A: Polym. Chem., 2019, 57, 1704-1710.

26 T. Thiounn, A. G. Tennyson and R. C. Smith, RSC Adv., 2019, 9, 31460-31465.

27 T. Thiounn and R. C. Smith, J. Poly. Sci., 2020, 58, 1347-1364.

28 M. S. Karunarathna and R. C. Smith, Sustainability, 2020, 12, 734-748.

29 M. S. Karunarathna, A. G. Tennyson and R. C. Smith, J. Mater. Chem. A, 2020, 8, 548-553.

30 C. V. Lopez, C. P. Maladeniya and R. C. Smith, Electrochemistry, 2020, 1, 226-259.

31 A. D. Smith, C. D. McMillin, R. C. Smith and A. G. Tennyson, J. Polym. Sci., 2020, 58, 438-445.

32 A. D. Smith, R. C. Smith and A. G. Tennyson, Sustain. Chem. Pharm., 2020, 16, 100249.

33 A. D. Smith, R. C. Smith and A. G. Tennyson, Sustain. Chem., 2020, 1, 209-237.

34 X. Zhang, Y. Tang, S. Qu, J. Da and Z. Hao, ACS Catal., 2015, 5, 1053-1067.

35 A. Demirbas, H. Alidrisi and M. A. Balubaid, Pet. Sci. Technol., 2015, 33, 93-101.

36 W. J. Chung, J. J. Griebel, E. T. Kim, H. Yoon, A. G. Simmonds, H. J. Ji, P. T. Dirlam, R. S. Glass,
J. J. Wie, N. A. Nguyen, B. W. Guralnick, J. Park, A. Somogyi, P. Theato, M. E. Mackay, Y.-E. Sung, K. Char and J. Pyun, Nat. Chem., 2013, 5, 518-524.

37 Y. Zhang, R. S. Glass, K. Char and J. Pyun, Polym. Chem., 2019, 10, 4078-4105.

38 T. S. Kleine, R. S. Glass, D. L. Lichtenberger, M. E. MacKay, K. Char, R. A. Norwood and J. Pyun, ACS Macro Lett., 2020, 9, 245-259.

39 P. Yan, W. Zhao, B. Zhang, L. Jiang, S. Petcher, J. A. Smith, D. J. Parker, A. I. Cooper, J. Lei and T. Hasell, Angew. Chem., Int. Ed., 2020, 59, 13371-13378.

40 M. J. H. Worthington, R. L. Kucera and J. M. Chalker, Green Chem., 2017, 19, 2748-2761.

41 J. M. Chalker, M. J. H. Worthington, N. A. Lundquist and L. J. Esdaile, Top. Curr. Chem., 2019, 377, 1-27.

42 A. G. Simmonds, J. J. Griebel, J. Park, K. R. Kim, W. J. Chung, V. P. Oleshko, J. Kim, E. T. Kim, R. S. Glass, C. L. Soles, Y.-E. Sung, K. Char and J. Pyun, ACS Macro Lett., 2014, 3, 229-232.

43 I. Gomez, O. Leonet, J. A. Blazquez and D. Mecerreyes, ChemSusChem, 2016, 9, 3419-3425.

44 A. Hoefling, D. T. Nguyen, Y. J. Lee, S.-W. Song and P. Theato, Mater. Chem. Front., 2017, 1, 1818-1822.

45 S. Zeng, L. Li, L. Xie, D. Zhao, N. Wang and S. Chen, ChemSusChem, 2017, 10, 3378-3386.

46 Y. Zhang, J. J. Griebel, P. T. Dirlam, N. A. Nguyen, R. S. Glass, M. E. MacKay, K. Char and J. Pyun, J. Polym. Sci., Part A: Polym. Chem., 2017, 55, 107-116.

47 P. Dong, K. S. Han, J.-I. Lee, X. Zhang, Y. Cha and M.-K. Song, ACS Appl. Mater. Interfaces, 2018, 10, 29565-29573.

48 Q. Jiang, Y. Li, X. Zhao, P. Xiong, X. Yu, Y. Xu and L. Chen, J. Mater. Chem. A, 2018, 6, 17977-17981.

49 F. Zhao, Y. Li and W. Feng, Small Methods, 2018, 2, 1-34.

50 T. S. Sahu, S. Choi, P. Jaumaux, J. Zhang, C. Wang, D. Zhou and G. Wang, Polyhedron, 2019, 162, 147-154.

51 J. J. Griebel, S. Namnabat, E. T. Kim, R. Himmelhuber, D. H. Moronta, W. J. Chung, A. G. Simmonds, K.-J. Kim, J. van der Laan, N. A. Nguyen, E. L. Dereniak, M. E. MacKay, K. Char, R. S. Glass, R. A. Norwood and J. Pyun, Adv. Mater., 2014, 26, 3014-3018.

52 S. Namnabat, J. J. Gabriel, J. Pyun and R. A. Norwood, Proc. SPIE, 2014, 8983, 89830D.

53 S. Namnabat, J. J. Gabriel, J. Pyun, R. A. Norwood, E. L. Dereniak and J. van der Laan, Proc. SPIE, 2014, 9070, 90702H.

54 J. J. Griebel, N. A. Nguyen, S. Namnabat, L. E. Anderson, R. S. Glass, R. A. Norwood, M. E. MacKay, K. Char and J. Pyun, ACS Macro Lett., 2015, 4, 862-866.

55 L. E. Anderson, T. S. Kleine, Y. Zhang, D. D. Phan, S. Namnabat, E. A. LaVilla, K. M. Konopka, L. Ruiz Diaz, M. S. Manchester, J. Schwiegerling, R. S. Glass, M. E. Mackay, K. Char, R. A. Norwood and J. Pyun, ACS Macro Lett., 2017, 6, 500-504.

56 T. S. Kleine, T. Lee, K. J. Carothers, M. O. Hamilton, L. E. Anderson, L. Ruiz Diaz, N. P. Lyons, K. R. Coasey, 
W. O. Parker, Jr., L. Borghi, M. E. MacKay, K. Char, R. S. Glass, D. L. Lichtenberger, R. A. Norwood and J. Pyun, Angew. Chem., Int. Ed., 2019, 58, 17656-17660.

57 J. Kuwabara, K. Oi, M. M. Watanabe, T. Fukuda and

T. Kanbara, ACS Appl. Polym. Mater., 2020, 2, 5173-5178.

58 T. Hasell, D. J. Parker, H. A. Jones, T. McAllister and

S. M. Howdle, Chem. Commun., 2016, 52, 5383-5386.

59 W. Thielke Michael, A. Bultema Lindsey, D. Brauer Daniel, P. Theato, B. Richter and M. Fischer, Polymers, 2016, 8(7), 266.

60 D. J. Parker, H. A. Jones, S. Petcher, L. Cervini, J. M. Griffin, R. Akhtar and T. Hasell, J. Mater. Chem. A, 2017, 5, 11682-11692.

61 A. M. Abraham, S. V. Kumar and S. M. Alhassan, Chem. Eng. J., 2018, 332, 1-7.

62 H.-K. Lin, Y.-S. Lai and Y.-L. Liu, ACS Sustainable Chem. Eng., 2019, 7, 4515-4522.

63 Y. Chen, A. Yasin, Y. Zhang, X. Zan, Y. Liu and L. Zhang, Materials, 2020, 13, 632.

64 A. D. Tikoalu, N. A. Lundquist and J. M. Chalker, Adv. Sustainable Syst., 2020, 4, 1900111.

65 C. R. Westerman and C. L. Jenkins, Macromolecules, 2018, 51, 7233-7238.

66 C. Herrera, K. J. Ysinga and C. L. Jenkins, ACS Appl. Mater. Interfaces, 2019, 11, 35312-35318.

67 K. Orme, A. H. Fistrovich and C. L. Jenkins, Macromolecules, 2020, 53, 9353-9361.

68 X. Wu, J. A. Smith, S. Petcher, B. Zhang, D. J. Parker, J. M. Griffin and T. Hasell, Nat. Commun., 2019, 10, 10035-10044.

69 B. Zhang, S. Petcher and T. Hasell, Chem. Commun., 2019, 55, 10681-10684.

70 B. Zhang, H. Gao, P. Yan, S. Petcher and T. Hasell, Mater. Chem. Front., 2020, 4, 669-675.

71 N. A. Lundquist, A. D. Tikoalu, M. J. H. Worthington, R. Shapter, S. J. Tonkin, F. Stojcevski, M. Mann, C. T. Gibson, J. R. Gascooke, A. Karton, L. C. Henderson, L. J. Esdaile and J. M. Chalker, Chem. - Eur. J., 2020, 26, 10035-10044.

72 S. J. Tonkin, C. T. Gibson, J. A. Campbell, D. A. Lewis, A. Karton, T. Hasell and J. M. Chalker, Chem. Sci., 2020, 11, 5537-5546.

73 M. P. Crockett, A. M. Evans, M. J. H. Worthington, I. S. Albuquerque, A. D. Slattery, C. T. Gibson, J. A. Campbell, D. A. Lewis, G. J. L. Bernardes and J. M. Chalker, Angew. Chem., Int. Ed., 2016, 55, 1714-1718.

74 F. Wu, S. Chen, V. Srot, Y. Huang, S. K. Sinha, P. A. van Aken, J. Maier and Y. Yu, Adv. Mater., 2018, 30(13), 1706643.

75 J. A. Smith, S. J. Green, S. Petcher, D. J. Parker, B. Zhang, M. J. H. Worthington, X. Wu, C. A. Kelly, T. Baker, C. T. Gibson, J. A. Campbell, D. A. Lewis, M. J. Jenkins, H. Willcock, J. M. Chalker and T. Hasell, Chem. - Eur. J., 2019, 25, 10433-10440.

76 J. E. M. Ballesteros, V. dos Santos, G. Marmol, M. Frias and J. Fiorelli, Cellulose, 2017, 24, 2275-2286.

77 R. P. Da Fonseca, J. C. Rocha and M. Cheriaf, Materials, 2021, 14, 155.
78 S. R. Ferreira, F. d. A. Silva, P. R. L. Lima and R. D. Toledo Filho, Constr. Build. Mater., 2017, 139, 551-561.

79 H. Fu, W. Gao, B. Wang, J. Zeng, Z. Cheng, J. Xu and K. Chen, Cellulose, 2020, 27, 1327-1340.

80 C. Gong, J.-p. Ni, C. Tian and Z.-h. Su, Int. J. Biol. Macromol., 2021, 172, 573-579.

81 C.-H. Ham, H. J. Youn and H. L. Lee, BioResources, 2020, 15, 9197-9211.

82 M. N. F. Norrahim, H. Ariffin, M. A. Hassan, N. A. Ibrahim, W. M. Z. W. Yunus and H. Nishida, Int. J. Nanotechnol., 2019, 16, 668-679.

83 P. Posada, J. Velasquez-Cock, C. Gomez-Hoyos, A. M. Serpa Guerra, S. V. Lyulin, J. M. Kenny, P. Ganan, C. Castro and R. Zuluaga, Cellulose, 2020, 27, 10649-10670.

84 A. Santmarti, T. Tammelin and K.-Y. Lee, Carbohydr. Polym., 2020, 250, 116870.

85 V. Vilchez, E. Dieckmann, T. Tammelin, C. Cheeseman and K.-Y. Lee, ACS Sustainable Chem. Eng., 2020, 8, 14263-14267.

86 C. Palange, M. A. Johns, D. J. Scurr, J. S. Phipps and S. J. Eichhorn, Cellulose, 2019, 26, 9645-9659.

87 X. Yang, E. Bakaic, T. Hoare and E. D. Cranston, Biomacromolecules, 2013, 14, 4447-4455.

88 M. S. Reid, M. Villalobos and E. D. Cranston, Langmuir, 2017, 33, 1583-1598.

89 S. J. Eichhorn, A. Dufresne, M. Aranguren, N. E. Marcovich, J. R. Capadona, S. J. Rowan, C. Weder, W. Thielemans, M. Toman, S. Renneckar, W. Gindl, S. Veigel, J. Keckes, H. Yano, K. Abe, M. Nogi, A. N. Nakagaito, A. Mangalam, J. Simonsen, A. S. Benight, A. Bismarck, L. A. Berglund and T. Peijs, J. Mater. Sci., 2010, 45, 1-33.

90 M. Mariano, N. El Kissi and A. Dufresne, J. Polym. Sci., Part B: Polym. Phys., 2014, 52, 791-806.

91 C. W. Wright, J. Chem. Educ., 1946, 23, 268-270.

92 S. P. Massie, Chemistry, 1970, 43, 18-21.

93 O. Zavgorodnya, J. L. Shamshina, P. Berton and R. D. Rogers, ACS Symp. Ser., 2017, 1250, 17-33.

94 M.-A. Perea-Moreno, F. Manzano-Agugliaro, Q. HernandezEscobedo and A.-J. Perea-Moreno, Sustainability, 2018, 10, 3254.

95 K.-H. Kim, J. L. Ong and O. Okuno, J. Prosthet. Dent., 2002, 87, 642-649.

96 S. Lin-Gibson, L. Sung, A. M. Forster, H. Hu, Y. Cheng and N. J. Lin, Acta Biomater., 2009, 5, 2084-2094.

97 T. Sakai, H. Li, T. Abe, S. Yamaguchi and S. Imazato, Dent. Mater., 2021, 37, 168-174.

98 J. D. Satterthwaite, A. Maisuria, K. Vogel and D. C. Watts, Dent. Mater., 2012, 28, 609-614.

99 G. E. Padawer and N. Beecher, Polym. Eng. Sci., 1970, 10, 185-192.

100 J. M. Adams, Clay Miner., 1993, 28, 509-530.

101 Additives for Plastics Handbook, ed. J. Murphy, 2nd edn, 2001.

102 Z.-L. He, L.-N. Chen, L. Zhang, H.-Y. Ren, M.-D. Xu and Y.-W. Lou, J. Appl. Polym. Sci., 2020, 137, 49010.

103 M. K. Lauer, M. S. Karunarathna, A. G. Tennyson and R. C. Smith, Mater. Adv., 2020, 1, 590-594.

104 S. Samal, S. Kim and H. Kim, J. Am. Ceram. Soc., 2012, 95, 1595-1603. 